\title{
Left hepatectomy in a patient with a Fontan circulation
}

\author{
Kevin Shing Lo ${ }^{1}$, Miu-Yee Chan ${ }^{2}$, Ka-Wing Ma ${ }^{2}$, Simon Hing-Yin Tsang ${ }^{2}$, Tan-To Cheung ${ }^{2}$, \\ Chung-Mau Lo ${ }^{2}$ \\ ${ }^{1}$ Department of Anaesthesiology, ${ }^{2}$ Department of Surgery, The University of Hong Kong, Hong Kong, China \\ Correspondence to: Dr. Tan-To Cheung. Chief of Hepatobiliary and Pancreatic Surgery, Department of Surgery, The University of Hong Kong, Queen \\ Mary Hospital, 102 Pokfulam Road, Hong Kong, China. Email: tantocheung@hotmail.com.
}

\begin{abstract}
Patients with a Fontan circulation face the long term risk of cardiac cirrhosis and the subsequent development of hepatocellular carcinoma (HCC). A hepatectomy operation imposes significant risk on such patients as the Fontan circulation can be severely compromised. Here we present a 24-year-old woman postFontan operation who successfully underwent a left hepatectomy, and discuss the anaesthetic and surgical management.
\end{abstract}

Keywords: Hepatocellular carcinoma (HCC); survival analysis; liver cancer; cirrhosis; inflow control; Pringle maneuver

Received: 04 July 2018; Accepted: 23 July 2018; Published: 02 August 2018.

doi: $10.21037 / \operatorname{tgh} .2018 .07 .10$

View this article at: http://dx.doi.org/10.21037/tgh.2018.07.10

\section{Introduction}

The Fontan operation was first described by Dr F. Fontan in 1971. It is a palliative procedure for patients with congenital heart defects resulting in a single ventricle physiology. The procedure separates the pulmonary circulation from the systemic circulation and effectively places them in series with the one functional ventricle. Consequently, flow through the pulmonary capillary bed becomes a completely passive process, dependent on a pressure gradient.

Medical advancement has improved the life expectancy of this palliative procedure. In long term survivors, the three leading causes of death are thromboembolism, heart failure, and sudden death-presumably from cardiac arrhythmias (1). The elevated systemic venous pressure of a Fontan circulation also exposes long-term survivors to the risk of cardiac cirrhosis and hepatocellular carcinoma (HCC). Management of this group of young adult with HCC imposes challenges to both surgeons and anaesthesiologists due to the unique haemodynamics.

\section{Case presentation}

Ms. K was born with tricuspid atresia, pulmonary valve atresia, univentricular heart and a patent ductus arteriosus.
At 51 weeks of age, she underwent a modified Fontan procedure (right atrium to main pulmonary artery connection) and ligation of a patent ductus arteriosus.

Ms. K subsequently underwent regular cardiac follow-up. At 19 years of age she complained of occasional palpitations. A 24-hour-Holter monitor detected only non-sustained atrial tachycardia which was not inducible during a later treadmill investigation. Ms. K refused thromboembolic prophylaxis with either warfarin or aspirin. Otherwise she enjoyed good health, completed secondary education, and had an exercise tolerance of three flights of stairs.

During her first surveillance abdominal ultrasound when she was 24 years old, a lobulated mass in segment 3 of her liver was detected. Her liver and renal function tests were normal with Child's A cirrhosis. Blood tests showed evidence of hypersplenism compatible with the imaging finding of cirrhosis and splenomegaly. The platelet count was $104 \times 10^{9} / \mathrm{L}$. International normalized ratio was 1.3. Alpha fetoprotein level was over $50,000 \mathrm{ng} / \mathrm{dL}$. Both hepatitis $\mathrm{B}$, hepatitis $\mathrm{C}$ and autoimmune markers were all negative. Indocyanine green retention test was performed and was $16.5 \%$ at 15 minutes.

The subsequent contrast computed tomography (CT) scan showed a cirrhotic liver with a large left lobe tumour. The tumour measured $6.8 \times 6.6 \times 5.1 \mathrm{~cm}$ with arterial 

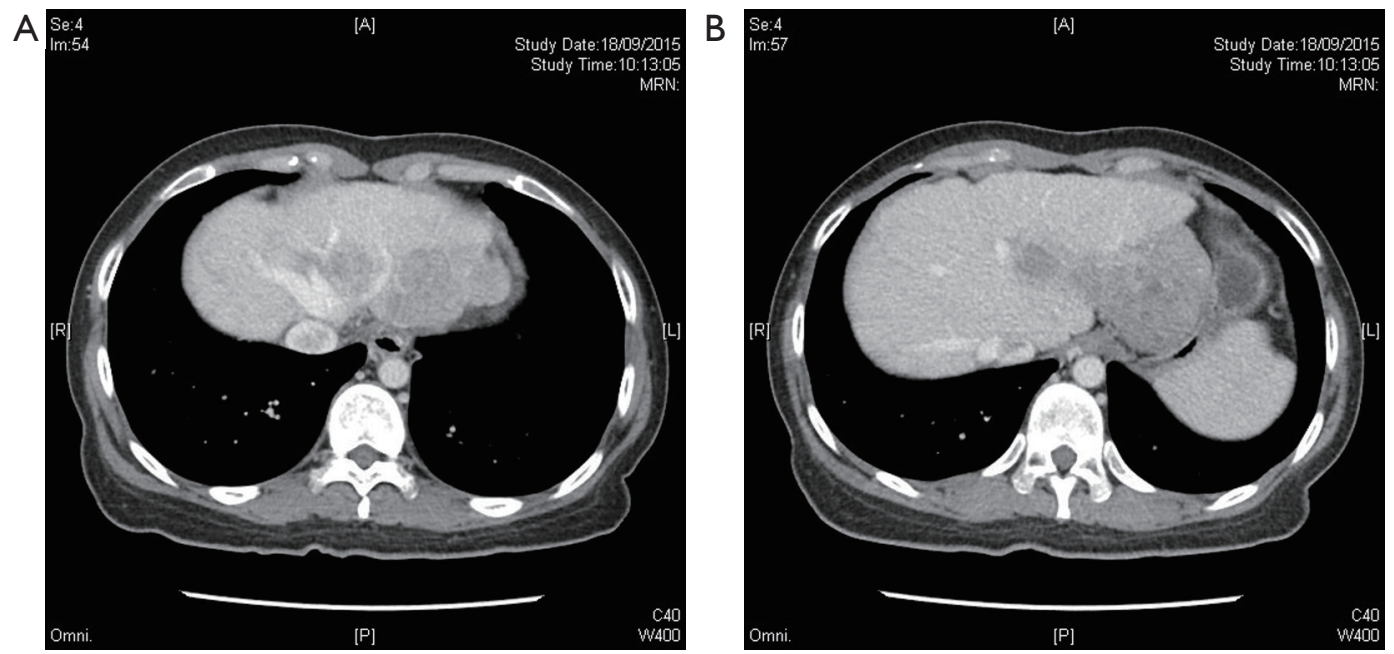

Figure 1 Pre-operative CT of the patient. (A) Close proximity of the tumour to left hepatic vein and abutting middle hepatic vein; (B) large exophytic tumour occupying left lobe). CT, computed tomography.

enhancement and portal venous washout (Figure 1). There was another smaller tumour in segment III measuring $2.7 \times 2.4 \times 1.9 \mathrm{~cm}$ contiguous with the larger tumour. The main portal vein and hepatic veins were still patent. Dual tracer (F-18 fluorodeoxyglucose and C-11 acetate) positron emission tomography (PET) scan was performed to rule out any extrahepatic lesion in view of the advanced tumour. It showed multifocal HCC with the dominant mass arising from the left lobe of liver, namely over segment II/IVA and segment $\mathrm{V}$. There was no distant metastasis. Upper endoscopy was also performed as the tumour was closely abutting the stomach, but there was no evidence of invasion.

\section{Pre-operative assessment}

An open extended left hepatectomy was planned therefore thorough cardiac assessment was undertaken. Physical examination showed no sign of congestive heart failure. Chest was clear and there were dual heart sounds with no S2 splitting. Oxygen saturation in room air was $93-98 \%$ with a respiratory rate of 14 per minute. Non-invasive blood pressure was 103-95/66-50 mmHg. She was ASA 3 according to American Society of Anaesthesiologist Classification and Class 2 according to the New York Heart Association Functional Classification.

Chest radiography showed clear lung fields and a globular heart. Heart size was normal but with a prominent right heart border and concave diaphragmatic border. 12lead ECG showed a sinus rhythm of $59 \mathrm{bpm}$. There was first-degree atrioventricular block and normal axis. There was bifid $\mathrm{p}$ wave in lead II, and $\mathrm{T}$ wave inversion in leads II, III, aVF, and V3-V5. Twenty-four-hour Holter monitoring detected 8 isolated premature ventricular complexes and infrequent 13 short runs of non-sustained atrial tachycardia (longest 60 beats at $94 \mathrm{bpm}$ ). Mean heart rate was $71 \mathrm{bpm}$ (45-104 bpm).

As part of her preoperative cardiac assessment we reviewed her previous cardio-pulmonary exercise test and transthoracic echocardiography, and performed a cardiac catheterisation. Her latest echocardiography showed no significant right atrial (RA) dilatation $(4.32 \times 3.95 \mathrm{~cm})$ or intracardiac thrombus. The left ventricle (LV) was mildly dilated. Left ventricular diastolic dimension was $5.5 \mathrm{~cm}$ with satisfactory function.

The latest cardiopulmonary exercise test showed peak heart rate of 136 beats per min (bpm) over a duration of 9.07 minutes. Peak $\mathrm{VO}_{2}$ was $21.9 \mathrm{~mL} / \mathrm{kg} / \mathrm{min}$, equivalent to 6 metabolic equivalents (METS) (1 METS $=3.5 \mathrm{~mL} / \mathrm{kg} / \mathrm{min}$ ). Around $60 \%$ of a female at her age (fair $=31-35 \mathrm{~mL} / \mathrm{kg} / \mathrm{min}$ ) or a fair 60 -year-old female. The peak minute ventilation was $33.4 \mathrm{~L} / \mathrm{min}$, and the respiratory exchange ratio was 1 . However the anaerobic threshold was not measured.

Cardiac catheterisation was performed to accurately assess the heart function. Left ventricular ejection function was satisfactory up to $58 \%$. Left ventricular end-diastolic pressure was $6 \mathrm{mmHg}$. There was dilated aortic root with no aortic insufficiency or major collaterals. The right atrium was dilated with a patent atriopulmonary connection. 


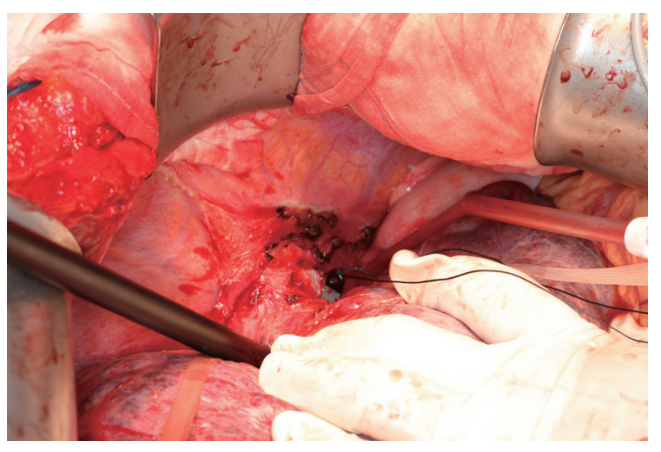

Figure 2 Isolating suprahepatic inferior vena cava.

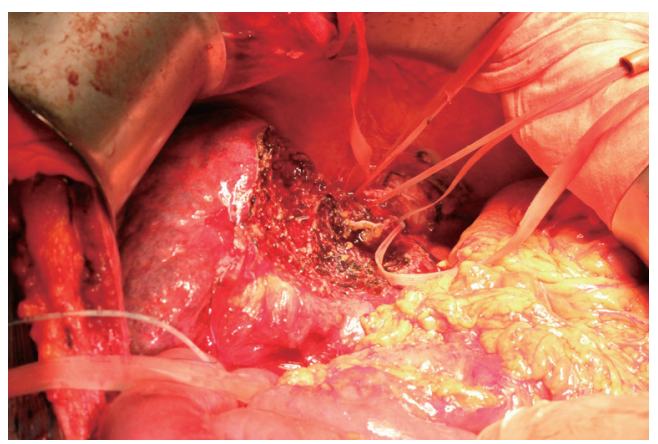

Figure 3 Remnant liver after transection. Hepatic veins were slung individually throughout the transection to accomplish total vascular exclusion.

There was no pulmonary artery stenosis and the size was satisfactory. Mean pressure of pulmonary artery, right atrium, superior and inferior vena cava was $9 \mathrm{mmHg}$. The pulmonary vascular resistance was 0.77 Wood Units and the pulmonary vascular resistance index was 1.29 Wood Units $\mathrm{m}^{2}$. The hepatic vein wedge pressure was $12 \mathrm{mmHg}$. The free hepatic venous pressure was $9 \mathrm{mmHg}$ and the hepatic venous pressure gradient was $3 \mathrm{mmHg}$. Fick right heart cardiac index was $3.8 \mathrm{~L} / \mathrm{min} / \mathrm{m}^{2}$ and the cardiac output was $6.3 \mathrm{~L} / \mathrm{min}$. Overall there was satisfactory haemodynamics with a patent Fontan circuit and no stenosis.

After several multidisciplinary meetings involving adult congenital cardiology, cardiothoracic surgery, hepatobiliary/ transplant surgery and anaesthesiology, various treatment options were explored. She was deemed not eligible for combined cardiac and liver transplantation due to her advanced tumour status. Her best chance of cure was still resection. However the operation also comprised of high morality risk up to $50-60 \%$ if the Fontan circulation was compromised. After thorough discussion with the patient and her family members, the patient agreed to proceed to operation.

\section{Operative procedure}

Standby extracorporeal membrane oxygenation (ECMO) was arranged with the help of cardiothoracic surgeons, as any chest compression or cardiac massage would be ineffective because of lack of valves to direct blood flow forward across pulmonary circulation. Anaesthesia was maintained with sevoflurane at 0.6-1 MAC, 36-48\% oxygen, and fentanyl infusion $1.5 \mathrm{microgram} / \mathrm{kg} / \mathrm{hr}$ and intermittent boluses of morphine (12 $\mathrm{mg}$ total) and ketamine $(10 \mathrm{mg}$ total). Muscle paralysis was maintained with a cisatracurium infusion $2-4 \mathrm{mg} / \mathrm{hr}$. Adrenaline infusion was commenced at $0.01 \mathrm{microgram} / \mathrm{kg} / \mathrm{min}$ in view of the transient hypotension after induction. Central venous catheter and Swan-Ganz catheter were placed. A transesophageal echocardiogram (TEE) probe was inserted to monitor the RA, left atrial (LA) and LV size, volume and contractility.

After induction, the CVP was $14 \mathrm{cmH}_{2} \mathrm{O}$. We aimed at a CVP of $11 \mathrm{cmH}_{2} \mathrm{O}$ because of myocardial depressant and vasodilatory effects of anaesthesia.

Upon laparotomy and intra-operative ultrasound, the patient was found to have macronodular cirrhosis and tumour had grown to $10.8 \mathrm{~cm}$ in size. The tumour was still confined to the left lobe. The inferior mesenteric vein (IMV) was cannulated to measure the portal pressure. It was noted that the portal flow was severely diminished due to high hepatic venous pressure. Hepatic artery flow was strong.

All the inflow and outflow vessels were dissected and slung before starting parenchymal transection. (Figures 2,3) test clamping of the IVC resulted in the CVP dropping to $6 \mathrm{cmH}_{2} \mathrm{O}$ and significant hypotension of $65 \mathrm{mmHg}$ systolic pressure. Test clamp of hepatic inflow did not affect the CVP or blood pressure. All three hepatic veins were slung individually in order to control outflow without disturbing the IVC. Left portal vein and left hepatic artery were divided. Parenchymal transection was performed with ultrasonic aspirator under total vascular occlusion for five cycles, each cycle of 15 minutes duration with 10-minute intervals between cycles. The middle hepatic vein was preserved.

After the transection, the left splenorenal shunt was ligated to ensure positive portal inflow against the elevated CVP (Figure 4). The portal pressure elevated to $20 \mathrm{cmH}_{2} \mathrm{O}$ after modulation. 


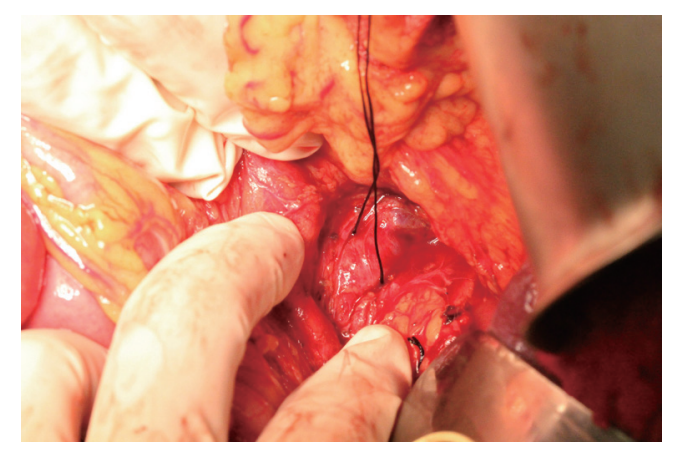

Figure 4 Ligation of splenorenal shunt.

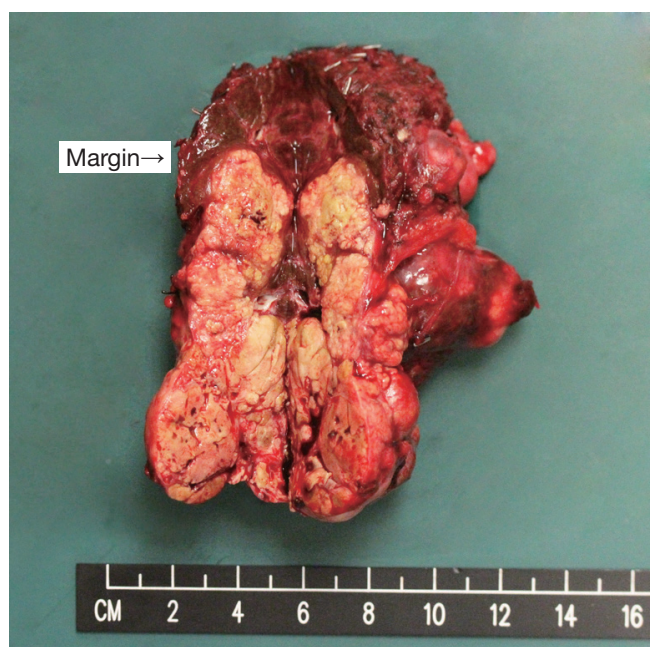

Figure 5 Specimen.

Towards the latter part of the operation, there was transient tachycardia and hypotension likely due to reperfusion of the liver after total vascular exclusion. On TEE the RA, LA and left ventricular filling were adequate; left ventricular contraction was good. No mitral or aortic valve regurgitation was detected. Nevertheless cardiac output with TEE was inaccurate. A fluid challenge raised the CVP to a mean of $14 \mathrm{mmHg}$ but there was no effect on blood pressure. Adrenaline infusion was thus increased and phenylephrine infusion was started. Ten units of red blood cell along with plasma, cryoprecipitate and platelets were given. Urine output was maintained at around $70 \mathrm{~mL} /$ hour. The operation lasted for 8.5 hours in total and blood loss was $4.1 \mathrm{~L}$.

Ms. K was extubated after the operation. Her blood pressure at the end of operation was $100 / 60 \mathrm{mmHg}$, heart rate was $130 \mathrm{bpm}$ and oxygen saturation was $98 \%$ on $2 \mathrm{~L} / \mathrm{min}$ of oxygen supplement. Haemoglobin level was $9.7 \mathrm{~g} / \mathrm{dL}$. INR was 1.9 and $\mathrm{CVP}$ was $14 \mathrm{cmH}_{2} \mathrm{O}$. There was no metabolic acidosis on arterial blood gas. She was sent to the intensive care unit with patient-controlled anaesthesia. No inotrope support was required upon leaving the operation theatre.

\section{Post-operative course}

Ms. K had an uneventful recovery after the operation. She was discharged back to general ward on post-operative day (POD) 5. The liver function improved gradually and she was able to be discharged home on POD 12. Pathology report revealed a moderately differentiated HCC with lymphovascular invasion and multiple tumour thrombi in the hilar vessels. Resection margin was clear (Figure 5). She was given 1 cycle of transarterial chemoembolization in view of the advanced tumour status.

She was seen regularly in the outpatient clinic with normalization of liver function. Unfortunately she was found to have tumour recurrence with invasion to portal vein 4 months after the operation. She was given targeted therapy as disease control and subsequently passed away 6 months after the operation.

\section{Discussion}

From the current literature, there are only isolated case reports about hepatectomy following Fontan operation (Table 1). Others mostly reported a more conservative approach including ablation, transarterial therapies and systemic treatment. This is by far the second report on patient undergoing major hepatectomy after Fontan procedure. When the liver function and fitness of the patient allows, operative treatment still remains as a gold standard for treatment of HCC. Although challenging, hepatectomy should be contemplated whenever possible to help this group of young patients. Otherwise, if the tumour is still within criteria, combined heart and liver transplantation can be considered. Local-regional therapies are often limited by the presence of pacemakers, extrahepatic shunts and abnormal vasculature (5).

These long-term survivors were also bound to develop cardiac cirrhosis due to the long-standing elevated hepatic venous pressure. Nevertheless there is no consensus on surveillance in this group of patient. Screening interval and the age of commencement both has not yet been well established. Our patient was offered her first surveillance 
Table 1 Reported cases of HCC after Fontan procedure undergoing resection

\begin{tabular}{lcccc}
\hline Reference & Age & Sex & Tumour size (cm) & Treatment \\
\hline Weyker et al., $2014(2)$ & 23 & Female & 14.8 & Extended right hepatectomy \\
Kwon et al., 2015 (3) & 32 & Male & 4 & Segment 6 and 7 resection \\
Takuma et al., 2016 (4) & 29 & Female & 1.5 & Segment 4 wedge resection \\
Our case & 24 & Female & 10.8 & Left hepatectomy \\
\hline
\end{tabular}

HCC, hepatocellular carcinoma.

ultrasound at the age of 24 and unfortunately diagnosed to have an advanced HCC upon her first scan. It would be important to gather more data to construct a more comprehensive surveillance program in order to pick up tumours early. Previous studies also showed long-term survivors of a Fontan operation were at increased risk of thromboembolism, arrhythmias, progressive heart failure, protein losing enteropathy, and plastic bronchitis (1). Therefore these should be ruled out during the preoperative assessment.

The most important aspects of managing this patient were:

(I) the preoperative assessment and selection of the patient;

(II) multidisciplinary input;

(III) not compromising the Fontan circulation during the operative period.

Cardiopulmonary exercise testing, transthoracic echocardiography, 24-hour-Holter monitoring, CXR, ECG, and expert cardiology opinion played a vital role in determining this patient's fitness for surgery. Her cardiopulmonary exercise testing revealed a capacity of 6 METS, equivalent to a 60-year-old female in fair health. Patients without a Fontan circulation but with an equivalent exercise tolerance can usually undergo major hepatic resections safely. Cardiac catheterisation was vital for quantifying the actual pressures of the patient's current stable Fontan circulation. Based on these patientspecific "normal" values, we were able to recalibrate our interpretation of intra-operative pressure readings.

Intraoperatively, in the case Ms. K's Fontan circulation, optimising passive flow across the pulmonary vasculature required maximising the RA-LA hydrostatic pressure gradient and minimising the pulmonary vasculature resistance. The CVP was maintained at above $9 \mathrm{mmHg}$ by replacing blood loss and monitoring for inadvertent surgical IVC compression. It was important to communicate with anaesthesiologist throughout the operation as any twisting of IVC during mobilization may cause significant drop in blood pressure. It was also important to gain access to IVC and individual hepatic veins before transection began. Test clamping of IVC, hepatic veins and hepatic inflow allowed us to understand the actual haemodynamic changes beforehand.

The left hepatectomy was only made possible by intermittently isolating the liver from the systemic circulation. Occluding individual hepatic veins increased the technical difficulty of the operation but allowed the IVC to remain patent while limiting venous backflow into the liver. The Pringle manoeuvre is also an effective method of reducing blood loss (6). However, posthepatic reperfusion hypotension is a known complication. Ischemic preconditioning prior to the Pringle manoeuvre has been shown to mitigate but not eliminate reperfusion hypotension.

Indeed, operating on patients with a Fontan circulation is a great challenge to surgeons and anaesthesiologists. Ideally in any hepatectomy, CVP was kept as low as possible to minimize blood loss caused by back flow from hepatic veins. However in such patients, the CVP dictates cardiac output and they are extremely sensitive to hypovolaemia. Yet, it was also important to avoid hypervolemia and pathological RA distension, which could precipitate arrhythmias. Delicate control of intravascular volume along with precise surgical techniques to minimize blood loss was crucial. Operative techniques to minimize blood loss have been reported in previous studies (7).

During the operation it was also important to assess the portal flow and pressure. We must ensure adequate portal flow especially in cirrhotic liver. CT scan is a convenient way to look for any portosystemic shunts. By simply ligating some of the major shunts, portal flow can be improved significantly. It was also important to preserve the middle hepatic vein, as further ischaemia due to focal outflow obstruction may cause liver failure in such a cirrhotic patient (8). 
Other important measures to ensure a safe operation include reducing the use of myocardial depressing drugs (such as sevoflurane), minimizing pulmonary vascular resistance by adjusting the minute volume to avoid hypoxic vasoconstriction and hypercarbia, and reducing intrathoracic pressure (9). In a similar case by Weyker et al. (2), they described the use of inhaled nitric oxide to empirically lower the PVR. We also limited the use of phenylephrine infusion as we feared a pure alpha agonist would only increase the SVR, and by an excessive amount, impeding forward flow through the Fontan circulation.

The patient was extubated early. It was noted that it helped with maintaining the blood pressure. It was likely due to the combined effects of cessation of the volatile anaesthetic agent and the resumption of spontaneous respiration. By eliminating sevoflurane, any myocardial depression would have been reversed and thus increased her cardiac output. Cessation of IPPV allowed the generation of negative intrathoracic pressures and thus improving RA filling. Combined with the reduction in pulmonary vascular resistance, forward flow through her Fontan circulation would have increased.

\section{Conclusions}

Patients with post-Fontan cardiogenic cirrhosis and HCC are challenging to manage. Expertise in the management of patients with a Fontan circulation needs to be disseminated in order to help this group of young adults. A comprehensive surveillance program should be offered early to pick up HCC in its early stage. A multidisciplinary approach to assessment and detailed operative planning is vital.

\section{Acknowledgements}

None.

\section{Footnote}

Conflicts of Interest: The authors have no conflicts of interest

doi: $10.21037 /$ tgh.2018.07.10

Cite this article as: Lo KS, Chan MY, Ma KW, Tsang SH, Cheung TT, Lo CM. Left hepatectomy in a patient with a Fontan circulation. Transl Gastroenterol Hepatol 2018;3:51. to declare.

Informed Consent: We could not obtain written informed consent from the patient as she had already passed away at the time of writing of the report, and her family could not be reached. However, verbal consent to release clinical data and intra-operative materials for research purposes has been obtained prior to operation.

\section{References}

1. Khairy P, Fernandes SM, Mayer JE, et al. Long-term survival, modes of death, and predictors of mortality in patients with Fontan surgery. Circulation 2008;117:85-92.

2. Weyker PD, Webb CA-J, Emond JC, et al. Anesthetic implications of extended right hepatectomy in a patient with fontan physiology. A A Case Rep 2014;2:99-101.

3. Kwon S, Scovel L, Yeh M, et al. Surgical management of hepatocellular carcinoma after Fontan procedure. J Gastrointest Oncol 2015;6:E55-E60.

4. Takuma Y, Fukada Y, Iwadou S, et al. Surgical Resection for Hepatocellular Carcinoma with Cardiac Cirrhosis after the Fontan Procedure. Intern Med 2016;55:3265-72.

5. Asrani SK, Warnes CA, Kamath PS. Hepatocellular Carcinoma after the Fontan Procedure. N Engl J Med 2013;368:1756-7.

6. Chouker A, Schachtner T, Schauer R, et al. Effects of Pringle manoeuvre and ischaemic preconditioning on haemodynamic stability in patients undergoing elective hepatectomy: a randomized trial. Br J Anaesth 2004;93:204-11.

7. Fan S-T, Lo C-M, Liu C-L, et al. Hepatectomy for hepatocellular carcinoma: toward zero hospital deaths. Ann Surg 1999;229:322.

8. Arlt J, Wei W, Xie C, et al. Modulation of hepatic perfusion did not improve recovery from hepatic outflow obstruction. BMC Pharmacol Toxicol 2017;18:50.

9. Dorsey D, Kwon S, Krieger E, et al. Conflicting Hemodynamic Goals in an Adult Patient With Fontan Physiology Presenting for Resection of a Hepatocellular Carcinoma. J Cardiothorac Vasc Anesth 2016;30:452-4. 